\title{
Neuroanatomical Profiles of Deafness in the Context of Native Language Experience
}

\author{
Olumide A. Olulade, Daniel S. Koo, Carol J. LaSasso, and Guinevere F. Eden \\ Center for the Study of Learning, Georgetown University Medical Center, Washington, District of Columbia 20057, and Center for Visual Language and \\ Visual Learning, Gallaudet University, Washington, District of Columbia 20002
}

The study of congenitally deaf adult humans provides an opportunity to examine neuroanatomical plasticity resulting from altered sensory experience. However, attributing the source of the brain's structural variance in the deaf is complicated by the fact that deaf individuals also differ in their language experiences (e.g., sign vs spoken), which likely influence brain anatomy independently. Although the majority of deaf individuals in the United States are born to hearing parents and are exposed to English, not American Sign Language (ASL) as their first language, most studies on deafness have been conducted with deaf native users of ASL (deaf signers). This raises the question of whether observations made in deaf signers can be generalized. Using a factorial design, we compared gray (GMV) and white (WMV) matter volume in deaf and hearing native users of ASL, as well as deaf and hearing native users of English. Main effects analysis of sensory experience revealed less GMV in the deaf groups combined (compared with hearing groups combined) in early visual areas and less WMV in a left early auditory region. The interaction of sensory experience and language experience revealed that deaf native users of English had fewer areas of anatomical differences than did deaf native users of ASL (each compared with their hearing counterparts). For deaf users of ASL specifically, WMV differences resided in language areas such as the left superior temporal and inferior frontal regions. Our results demonstrate that cortical plasticity resulting from deafness depends on language experience and that findings from native signers cannot be generalized.

Key words: deafness; language; native users of American Sign Language; native users of English; VBM

\section{Introduction}

Long-term experience affects brain anatomy. For example, vocational skills honed by taxi drivers (Maguire et al., 2000) foster greater gray matter volume (GMV) in regions subserving this skill, as do educational experiences such as written language acquisition (Carreiras et al., 2009). Conversely, early blindness results in gray matter reduction in visual areas (Noppeney et al., 2005; Noppeney, 2007; Ptito et al., 2008). Deafness, however, is more difficult to assess because altered sensory experience in deaf individuals is concomitant with altered language experience (e.g., a lifetime experience with sign language). Therefore, it is challenging to make attributions solely to auditory deprivation. In the case of deaf users of sign language, differentiation of sensory and

\footnotetext{
Received Aug. 29, 2013; revised Feb. 24, 2014; accepted March 17, 2014

Author contributions: D.S.K., C.J.L., and G.F.E. designed research; 0.A.O., D.S.K., C.J.L., and G.F.E. performed research; 0.A.O. analyzed data; 0.A.O., D.S.K., C.J.L., and G.F.E. wrote the paper.

The authors declare no competing financial interests.

This work was supported by the Eunice Kennedy Shriver National Institute of Child Health and Human Development (Grant P50 HD40095), the National Institute on Deafness and Other Communication Disorders (Grant F32 DC007774), the National Science Foundation (Grant SBE 0541953), and a supplement from the National Institutes of Health and the National Science Foundation (Grant SBE 0541953). We thank Eileen Napoliello, Kelly Lamar Crain, Jenni Rosenberg, lain DeWitt, Alina Engelman, Megan Luetje, and Jill Weisberg for aiding with data collection and our participants for their involvement in the study. We also thank the staff at the (enter for Functional and Molecular Imaging, and the support of the Intellectual and Development Disorders Research (enter (IDDRC) Grant 5P3OHD040677-13.

Correspondence should be addressed to Guinevere F. Eden, D. Phil., Georgetown University Medical Center, Building D, Suite 150, 4000 Reservoir Road NW, Washington, DC 20057. E-mail: edeng@georgetown.edu.

DOI:10.1523/JNEUROSCI.3700-13.2014

Copyright $\odot 2014$ the authors $\quad 0270-6474 / 14 / 345613-08 \$ 15.00 / 0$
}

language experience has traditionally been addressed by the inclusion of hearing participants who sign from birth, thus sharing the deaf participants' experience of a visuospatial language (Allen et al., 2008, 2013).

It is often assumed that all deaf individuals grow up using sign language. In reality, most deaf individuals (95\%) in America are born to hearing parents (Mitchell and Karchmer, 2004) and, instead of a sign language, most of these learn English as a first language (L1) via lip/speech reading; indeed, $>80 \%$ of American deaf students report regular use of English in their homes and schools (Gallaudet Research Institute, 2010). However, such a group of deaf individuals has not been included in prior investigations into the neuroanatomical basis of deafness. This is a notable omission, not only because these deaf native users of English are more representative of the deaf population than the small fraction born to deaf parents, but also because their inclusion provides a good comparison group for hearing subjects, bypassing native sign language experience.

Previous studies examining anatomical effects of deafness (Emmorey et al., 2003; Penhune et al., 2003; Fine et al., 2005; Kara et al., 2006; Shibata, 2007; Allen et al., 2008, 2013; Kim et al., 2009; Smith et al., 2011) have achieved limited convergence. Whereas studies of blind individuals reliably observe visual cortex gray matter atrophy (Noppeney, 2007, but see Bridge et al., 2009), there have been no analogous reports in auditory cortex in deaf individuals. Although there are findings of less white matter in auditory regions (Emmorey et al., 2003; Shibata, 2007; Smith et 
Table 1. Participant demographic information

\begin{tabular}{|c|c|c|c|c|c|c|c|c|}
\hline & \multicolumn{3}{|c|}{ Native users of ASL } & \multicolumn{3}{|c|}{ Native users of English } & \multicolumn{2}{|c|}{ ANOVA $^{a}$} \\
\hline & Deaf & Hearing & $p$ & Deaf & Hearing & $p$ & $F$ & $p$ \\
\hline F/M & $8 / 7$ & $12 / 3$ & - & $5 / 10$ & $7 / 8$ & - & - & - \\
\hline Range & $18.4-31.8$ & $18.4-39.5$ & & $22.8-34.6$ & $18.6-41.8$ & & & \\
\hline \multicolumn{9}{|l|}{ PIQ } \\
\hline Mean \pm SD & $112.0 \pm 9.8$ & $113.2 \pm 11$ & NS & $113.3 \pm 8.9$ & $118.4 \pm 7.6$ & NS & 1.44 & NS \\
\hline Mean \pm SD & $107.6 \pm 16$ & $106.7 \pm 13$ & NS & $119.5 \pm 11$ & $110.3 \pm 17$ & NS & 2.49 & NS \\
\hline Range & 89-131 & $76-121$ & & 95-131 & 75-131 & & & \\
\hline
\end{tabular}

${ }^{a}$ One-way ANOVA including all four groups. NS, Nonsignificant $(p>0.05)$.

al., 2011), gray matter has been shown to be the same (Penhune et al., 2003) and even larger in deaf relative to hearing groups (Emmorey et al., 2003; Smith et al., 2011).

Here, we test the prediction that lifetime absence of auditory input is reflected in less GMV and white matter volume (WMV), specifically in early auditory regions. In the first study, we compared adult deaf and hearing native users of American Sign Language (ASL), testing this hypothesis in the signing population on which prior work has focused and providing consistency with existing publications. Then, we expanded the study to deaf and hearing adults who report English (not ASL) as their native language, thus testing the effects of deafness in a factorial design that also considers native language experience.

\section{Materials and Methods}

Participants. In the first study, deaf native users of ASL (i.e., deaf individuals born to deaf parents, also referred to as "deaf signers") were compared with hearing native users of ASL (i.e., hearing individuals born to deaf parents, also referred to as "hearing signers"; in the literature, these individuals are also referred to as children of deaf adults [CODAs]). These two groups of signing participants are similar to those included in previous studies comparing deaf and hearing signers (Allen et al., 2008, 2013). For ease of comparison with these prior studies, GMV and WMV from deaf and hearing native signers were compared using a two-sample $t$ test. Just as in the prior work involving signers, the logic that applies here is that this comparison should reveal differences attributed to their contrasting auditory experiences because both have in common that they are native users of ASL (which they learned from birth from their signing parents). However, to expand on the previous studies, both of which had restricted their analyses to regions of interest (Allen et al., 2008, 2013), we used a whole-brain analysis.

In the second study, the primary focus of this investigation, we expanded the sample to include deaf native users of English (i.e., deaf individuals born to two hearing parents) and hearing native users of English (i.e., hearing individuals born to two hearing parents). These deaf participants self-reported English as their native language on a questionnaire completed before enrolling in the study. Therefore, we refer to this group as "deaf native users of English" because English is their first language (English L1), it is the language used in their homes and at school, and it was their only language through most of childhood and adolescence. Although many of these participants learned ASL as young adults, they still use English most of the time on a daily basis and it is their preferred language. Their access to English is through oral communication (lip-reading) or cued speech (i.e., augmenting lip reading with a series of hand motions and hand placements on the face that fully distinguish the phonemes of English and other traditionally spoken languages). GMV and WMV from all four groups were submitted to an ANOVA to test for differences specific to their sensory experience (deaf vs hearing), their language experience (ASL vs English), and the interactions of these.

All deaf individuals (deaf native users of ASL and deaf native users of English) had a severe hearing loss of at least $75 \mathrm{~dB}$ in the better ear based on prior clinical audiologic evaluation (conducted on average 18 months before the subject's participation in the study). Average hearing loss in the better ear was matched between the two deaf groups, with $87 \mathrm{~dB}$ loss for the deaf native users of ASL and $89 \mathrm{~dB}$ loss for the deaf native users of English. All of the deaf individuals were either congenitally deaf or became deaf before the age of two years. All participants were right handed, as assessed via the Edinburgh Handedness Inventory (Oldfield, 1971), and none reported any history of congenital or acquired neurological or psychological disorder.

All participants had completed at least a formal high school education and most were enrolled in college (at the undergraduate or graduate level) at the time of participation. All individuals were within or above the normal range (i.e., a score of 85 or above) for nonverbal intelligence as measured by the Wechsler Abbreviated Scale of Intelligence (WASI; Wechsler, 1999).

There was a total of 60 participants ( 32 female, 28 male), 15 participants in each of the four groups. Their demographic information is provided in Table 1. The four groups were equated on age, performance IQ (PIQ) assessed by the WASI (Wechsler, 1999), and single word reading measured by the Test of Silent Word Reading Fluency (TOSWRF; Mather et al., 2004; single-factor ANOVAs, $n=60$; main effect of group was nonsignificant; $p>0.05$ for all three measures). For consistency with the two-way ANOVA used for the neuroanatomical data (see "Image Analysis" section), these behavioral data were also submitted to an ANOVA examining main effects of sensory experience (deaf vs hearing), main effect of language experience (ASL vs English), and their interaction. This analysis revealed a significant main effect of sensory experience for the TOSWRF measure $\left(F_{(1,58)}=4.16 ; p=0.046\right)$ with an advantage observed for the deaf groups. To ensure that any findings were unrelated to this difference, the ANOVAs of GMV and WMV were performed with and without this measure included as a covariate of no interest. The results were largely the same and are reported here using the data with TOSWRF included as a covariate of no interest in the analysis.

The Georgetown University Institutional Review Board and the Gallaudet University Institutional Review Board granted approval of the study, and participants provided informed written consent for their participation.

Image acquisition. High-resolution T1-weighted MR images were acquired on a 3T Siemens Trio scanner located in the Center for Functional and Molecular Imaging at the Georgetown University Medical Center. Images consisted of 160 contiguous slices and were acquired with the following parameters: voxel size $=1 \mathrm{~mm} \times 1 \mathrm{~mm} \times 1 \mathrm{~mm}, \mathrm{TR}=1600$ $\mathrm{ms}, \mathrm{TE}=3.37 \mathrm{~ms}$, flip angle $=15^{\circ}, \mathrm{FOV}=256 \mathrm{~mm}$. Subjects were asked to remain as still as possible during the scan. Three or more highresolution images were acquired for most participants, depending on time constraints and participant compliance. In these cases, the images were inspected and blindly rated for motion artifacts by two individuals, with the best image chosen for inclusion in subsequent analysis.

Image analysis. Analysis of images consisted of volume measurements using the automated voxel-based morphometry (VBM) technique implemented in SPM8 (http://www.fil.ion.ucl.ac.uk/spm/) via the methods outlined by Ashburner and Friston (2000). Prior to preprocessing, the 


\section{Native Users of American Sign Language}

A Gray Matter Volume Hearing > Deaf

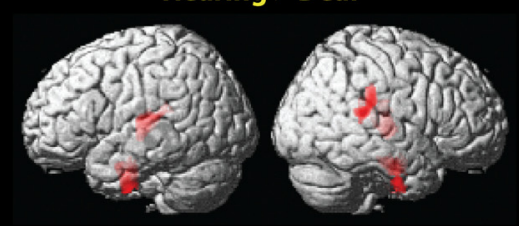

Deaf $>$ Hearing
B White Matter Volume

Hearing > Deaf

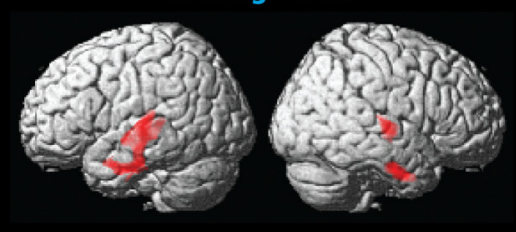

Deaf $>$ Hearing

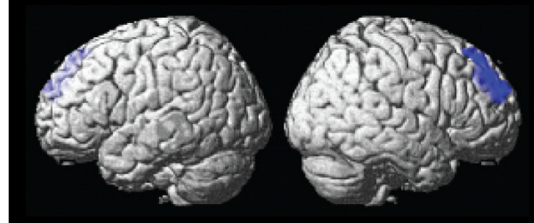

No Significant Clusters

Figure 1. GMV and WMV differences between deaf and hearing native users of ASL. Less GMV $(\boldsymbol{A})$ was observed in the deaf group in bilateral temporal lobe regions, including Heschl's gyrus. Less WMV $(\boldsymbol{B})$ was also observed in the bilateral temporal lobe regions, close to areas identified to differ in gray matter. Deaf signers had more GMV in right superior frontal cortex. Height threshold $p<0.005$; nonstationary corrected threshold $p<0.05$. Clusters were overlaid onto the standardized MNI brain template.

original images from all participants were manually realigned to the anterior commissure to reduce between-subject variability in spatial location. Images were subsequently processed via the following steps: coregistration to the white matter template; segmentation to gray matter, white matter, and CSF using the "New Segment" toolbox (Ashburner and Friston, 2005), registration of each image to a custom studyspecific template, and spatial normalization to the MNI stereotaxic space via affine registration of the generated template to the MNI template using DARTEL (Ashburner, 2007). In the previous step, the "modulation" option was selected so that observed differences represented tissue volume rather than density. Finally, images were smoothed with an isotropic Gaussian kernel of $10 \mathrm{~mm}$ FWHM to reduce anatomical variability and intensity was thresholded at 0.2 to reduce edge artifacts. Images were visually inspected to ensure accuracy of the spatial normalization procedure.

For statistical analysis, we performed a between-group $t$ test for the first study and an ANOVA for the second, both at the level of the whole brain. First, whole-brain between-group statistical analysis was implemented using SPM8 to compare GMV and WMV between deaf and hearing native users of ASL. For the spatial normalization procedure implemented during the preprocessing stage, the study-specific template was generated using only these two groups of participants (total $n=30$ ). Two-sample $t$ tests were conducted to reveal where deaf individuals exhibited greater GMV or WMV than hearing individuals and vice versa. Images were thresholded at a height level of $p<0.005$ (minimum cluster size $=95$ contiguous voxels) and nonstationary cluster corrected (Hayasaka et al., 2004) at a threshold of $p<0.05$. There were no between-group differences in total intracranial volume (TIV: the sum of whole-brain $\mathrm{GMV}, \mathrm{WMV}$, and CSF), but to be sure to infer regional variation in brain matter, this measure was included as a regressor of no interest in the analyses (Peelle et al., 2012).

In the second analysis, an ANOVA was implemented at the wholebrain level for all four groups using SPM8 to determine brain matter volume differences related to sensory experience (deaf vs hearing), language experience (ASL vs English), and interactions between the two. A new comparison-specific template was generated for the spatial normalization, this time using all subjects $(n=60)$. Gray and white matter images were entered into a $2 \times 2$ factorial design with sensory experience and language experience as between-subject factors. An uncorrected threshold of $p<0.005$ (minimum cluster size $=95$ contiguous voxels) was applied. Clusters surviving this threshold were extracted using Mars$\mathrm{BaR}$ (http://marsbar.sourceforge.net/) to examine the direction of ob- served effects. Again, TIV did not significantly differ between the four groups, but was entered as a covariate of no interest in this analysis to be conservative.

\section{Results}

Between-group comparisons of deaf and hearing native users of ASL

Hearing $>$ deaf

GMV. Greater GMV was observed for the hearing native signers relative to the deaf native signers within the left transverse temporal (Heschl's) gyrus, extending into the superior temporal gyrus and the insula (Fig. $1 A$, Table 2). In the right hemisphere, greater GMV was found for the hearing group within the claustrum and extending into the transverse and superior temporal gyri, the insula, and portions of the inferior parietal lobule. Greater GMV was also observed in the left middle temporal gyrus and in the right fusiform gyrus. In both hemispheres, these clusters extended into the inferior temporal, fusiform, and parahippocampal regions.

WMV. Areas with more WMV for the hearing group (Fig. 1B, Table 2) were located in close proximity to those observed for gray matter. A large cluster revealing greater WMV for the hearing signers relative to the deaf signers was observed in the left hemisphere, with a peak location in the middle temporal gyrus and extending into the superior, transverse, and inferior temporal gyri, as well as the insula, fusiform, and parahippocampal gyri. In the right hemisphere, more white matter in the hearing compared with the deaf signers was observed in the superior temporal gyrus, extending into Heschl's gyrus and the insula. A second right hemisphere region was observed in the middle temporal gyrus, extending into the fusiform and inferior temporal regions.

\section{Deaf $>$ hearing}

GMV. The opposite contrast (Fig. 1A, Table 2) yielded greater GMV in the deaf native signers compared with the hearing native signers in the right superior frontal gyrus, extending into the middle frontal gyrus.

WMV. No differences in WMV were observed between deaf and hearing native signers.

\section{ANOVA of deaf and hearing native users of ASL and deaf and} hearing native users of English

Main effect of sensory experience

Hearing $>$ deaf. For gray matter (Fig. $2 A$, top, Table 3), the two hearing groups combined compared with the two deaf groups combined exhibited greater GMV in the left anterior fusiform and lingual gyri and in the left cerebellum. Hearing groups also had greater GMV than the deaf groups in the right precuneus and in the right posterior cingulate.

For white matter (Fig. 2B, top, Table 3), the hearing groups overall had greater WMV than the deaf groups in the left superior temporal gyrus, extending into the transverse temporal (Heschl's) gyrus.

Deaf $>$ hearing. Relatively greater GMV was observed for the deaf groups in the middle temporal regions bilaterally, and in the bilateral superior frontal gyri, extending into the middle frontal gyrus in the right hemisphere.

There were no findings of greater WMV in the deaf groups relative to the hearing groups.

\section{Main effect of language experience}

$A S L>$ English. Deaf and hearing native users of ASL combined exhibited greater GMV than deaf and hearing native users of English, predominantly in right hemisphere regions. These included the right middle, medial, and inferior frontal cortices and the precuneus (Fig. $2 \mathrm{~A}$, middle, Table 3). Left hemisphere differences were observed in the middle frontal 
Table 2. MNI coordinates of maxima of volume differences for whole-brain between-group comparisons of deaf and hearing native users of ASL

\begin{tabular}{|c|c|c|c|c|c|c|}
\hline \multirow[b]{2}{*}{ Comparison } & \multicolumn{3}{|c|}{ MNI coordinates } & \multirow[b]{2}{*}{ Anatomical region } & \multirow[b]{2}{*}{ BA } & \multirow[b]{2}{*}{ Z } \\
\hline & $x$ & $y$ & $Z$ & & & \\
\hline \multirow[t]{9}{*}{ Hearing $>$ deaf } & GMV c & & & & & \\
\hline & -33 & -25 & 6 & L. transverse temporal gyrus ${ }^{a}$ & 41 & 4.01 \\
\hline & -36 & -3 & -30 & L. middle temporal gyrus ${ }^{b}$ & 21 & 4.61 \\
\hline & 32 & -18 & 12 & R. claustrum ${ }^{c}$ & & 3.92 \\
\hline & 39 & -6 & -27 & R. fusiform gyrus ${ }^{b}$ & 20 & 3.82 \\
\hline & WMV & & & & & \\
\hline & -52 & -22 & -8 & L. middle temporal gyrus ${ }^{d}$ & 21 & 4.93 \\
\hline & 50 & -21 & 9 & R. superior temporal gyrus ${ }^{e}$ & 41 & 4.00 \\
\hline & 46 & 2 & -38 & R. middle temporal gyrus ${ }^{f}$ & 21 & 4.04 \\
\hline \multirow[t]{4}{*}{ Deaf $>$ hearing } & GMV c & & & & & \\
\hline & 22 & 41 & 52 & R. superior frontal gyrus ${ }^{g}$ & 8 & 4.07 \\
\hline & WMV & & & & & \\
\hline & No sig & & & & & \\
\hline
\end{tabular}

${ }^{a}$ Cluster extends into the superior temporal gyrus and the insula.

${ }^{b}$ Cluster extends into the inferior temporal gyrus, the fusiform gyrus, and parahippocampal gyrus.

'Cluster extends into the transverse temporal gyrus, superior temporal gyrus, the insula, and the inferior parietal lobule.

${ }^{d}$ Cluster extends into the transverse temporal gyrus, superior temporal gyrus, inferior temporal gyrus, insula, fusiform gyrus, and parahippocampal gyrus.

${ }^{e}$ Cluster extends into the transverse temporal gyrus and insula.

${ }^{f}$ Cluster extends into the fusiform gyrus and inferior temporal gyrus.

${ }^{g}$ Cluster extends into the middle frontal gyrus.

gyrus and in the cingulate gyrus, where native users of ASL had more GMV than native users of English.

Greater WMV (Fig. 2B, middle, Table 3) was observed for native users of ASL in the left precentral gyrus and right inferior frontal gyrus.

English $>$ ASL. Deaf and hearing native users of English did not exhibit any areas of greater GMV or WMV compared with deaf and hearing native users of ASL.

Interaction: sensory experience by language experience

$G M V$. Significant findings for this interaction emerged in five areas (Fig. $2 A$, bottom, Table 3 ): left hemisphere middle temporal gyrus and caudate and right hemisphere medial frontal and precentral gyri and insula. The question central to this investigation is whether the differences in GMV in deaf compared with hearing users of sign are unique to deaf native signers, as evidenced by absence or reversal of this difference in the other pair, the deaf and hearing native users of English. The first type of pattern was observed in right insula, where the deaf native users of ASL had less GMV than the hearing native users of ASL, with GMV here being equal in the deaf and hearing native users of English. Right precentral gyrus also had less GMV in the deaf native users of ASL compared with the hearing native users of ASL; here the deaf native users of English showed more GMV compared with their hearing counterparts (i.e., the reverse of the signing groups). Less GMV in these two right hemisphere regions is therefore specific to deaf native users of sign.

Conversely, deaf native users of ASL had more GMV than their hearing counterparts in the right medial frontal gyrus and left caudate, whereas this observation was the reverse in the deaf compared with hearing native users of English. Together, these four differences in GMV (less in right precentral gyrus and insula; more in right medial frontal gyrus and left caudate) can be attributed to deafness only in native users of ASL.

The deaf native users of English showed, as just described, less GMV in right medial frontal gyrus and left caudate compared with their hearing native users of English comparison group. Also, as just described, there was more GMV in right precentral gyrus in deaf compared with hearing native users of English, whereas the effect was the opposite in the deaf and hearing native users of ASL. In addition, for the fifth cluster identified in the interaction analysis, more GMV was observed in the left middle temporal gyrus in the deaf compared with the hearing native users of English, whereas it was equal in the comparison of deaf and hearing native users of ASL. In fact, GMV in this region was greater in the deaf native users of English than in any of the three other groups. Together, less GMV in the right medial frontal gyrus and the left caudate and more GMV in the right precentral and left middle temporal gyri can be attributed specifically to deafness in native users of English.
$W M V$. The interaction analysis revealed five clusters (Fig. $2 B$, bottom, Table 3): left hemisphere superior temporal and inferior frontal gyri and cerebellum and right hemisphere precentral and fusiform gyri, extending into the middle and inferior temporal cortices. Again, our interest was primarily in areas where the deaf and hearing native users of ASL had a difference in WMV, whereas there was no difference or a reversal of the relationship between the deaf and hearing native users of English. This was the case in left superior temporal and inferior frontal gyri and in the right fusiform region, which were less in the deaf compared with the hearing native users of ASL (and equal in the deaf and hearing native users of English), suggesting that this experience-dependent difference in WMV attributed to deafness is unique to deaf people who are native signers, but cannot be considered generalizable to all deaf people. There were no areas where deaf native users of ASL had more WMV than their hearing counterparts.

The analysis also revealed that deaf native users of English have less WMV in the cerebellum than their hearing counterparts, but this was driven, as can be seen in Figure 2B, by more WMV in the hearing native users of English than in any of the other groups. Conversely, deaf native users of English had more WMV than their hearing counterparts in right precentral gyrus, whereas WMV was equal here in the deaf and hearing native signers. In sum, less WMV resulting from deafness is unique to native users of sign in left superior temporal gyrus, left inferior frontal gyrus, and right fusiform gyrus, whereas, in deaf native users of English, deafness manifests in less WMV in cerebellum and more WMV in right precentral gyrus.

\section{Discussion}

The present study investigated how lack of auditory experience affects brain anatomy by contrasting GMV and WMV in deaf and hearing adults using whole-brain voxel-based morphometry. By analogy with reports on visual system differences in studies of the blind, one might expect less brain volume in early auditory regions. However, studies of deafness are complicated by the fact that deaf individuals are raised with varying language experiences and acoustic stimuli and language are both processed in auditory regions within the superior temporal gyrus. Therefore, our study involved deaf and hearing participants with and without native sign experience to determine whether the effects of deafness manifest differently on brain matter in deaf individuals with distinct language backgrounds. 


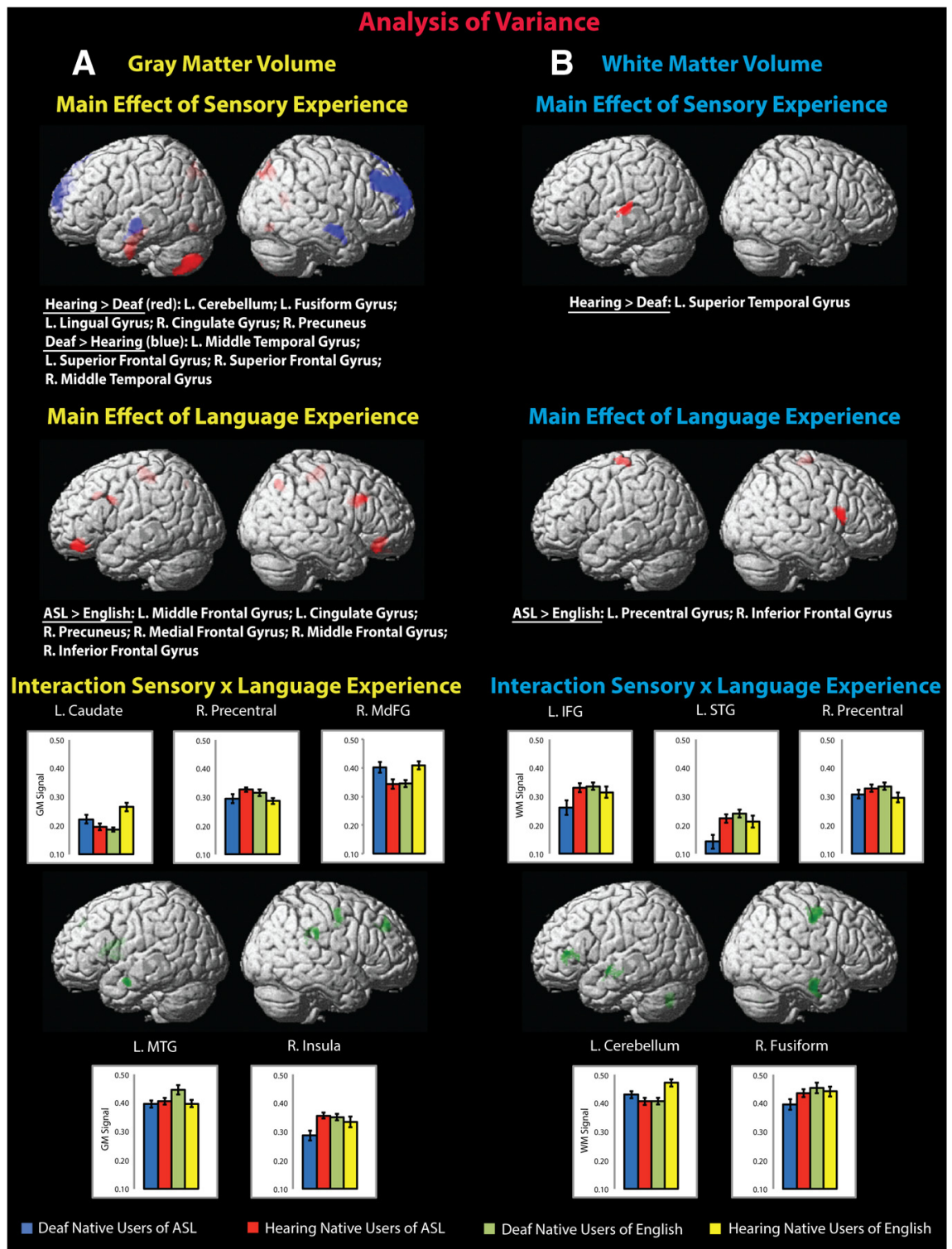

Figure 2. Results of ANOVA. Shown are the ANOVA results for GMV $(\boldsymbol{A})$ and WMV $(\boldsymbol{B})$ differences. Top, Main effect of sensory experience (hearing $>$ deaf, red; deaf $>$ hearing, blue). Middle, Main effect of language experience (ASL $>$ English, red). Bottom, Interaction of sensory experience $\times$ language experience (green). MdFG, medial frontal gyrus; IFG, inferior frontal gyrus; MTG, middle temporal gyrus; STG, superior temporal gyrus. Error bars indicate 1 SE measurement. Height threshold $p<0.005$; minimum cluster size $=95$. Clusters were overlaid onto the standard MNI brain template.

Our first study of deaf native signers was intended to provide consistency with prior investigations that have focused primarily on this population. Hearing native signers served as the comparison group because differences between deaf signers and hearing nonsigners as reported by most (but see Allen et al., 2008, 2013) make it impossible to make attributions to sensory versus language experience. Unlike prior work, in the present study, we investigated the whole brain. Prior work in the deaf (using hand tracing, VBM, and other methods) has often examined a priori regions of interest to focus specifically on auditory areas (Emmorey et al., 2003; Penhune et al., 2003; Smith et al., 2011), visual areas (Fine et al., 2005; Allen et al., 2013), insula (Allen et al., 2008), motor cortex and inferior frontal gyrus (Allen et al., 2013), and corpus callosum (Kara et al., 2006; Leporé et al., 2010). Those who examined differences at the whole-brain level have in some cases reported differences in auditory regions (Shibata, 2007;
Kim et al., 2009; Leporé et al., 2010; Pénicaud et al., 2012) and elsewhere, including parietal, frontal, and temporal lobes; motor regions; and cerebellum (Penhune et al., 2003; Shibata, 2007; Kim et al., 2009; Leporé et al., 2010). When comparing deaf with hearing native users of ASL across the whole brain, we found less GMV and WMV in superior temporal regions bilaterally, including primary auditory cortex. Less WMV in auditory brain regions has been reported in comparisons of adult deaf signers and hearing nonsigners (Emmorey et al., 2003; Shibata, 2007; Leporé et al., 2010; Pénicaud et al., 2012), and deaf and hearing prelingual infants (Smith et al., 2011). Our replication of less WMV in superior temporal regions bilaterally, achieved here with a hearing native signing control group, is consistent with work conducted without hearing signers; it also converges with our novel finding of less GMV in bilateral transverse temporal gyri. An interesting outcome of this analysis is the finding of more GMV in the deaf native signers in right superior frontal cortex.

Our second and main analysis served to address the concern that prior studies have largely involved deaf native users of ASL, who represent a small section of the deaf population and whose native experience with a spatial language might play a mitigating role in the results to date (Cardin et al., 2013). Although both the hearing and deaf participants in the first study were matched on their ASL experience, the experience of a signed language might add another layer of experience-induced plasticity that could in part be responsible for the anatomical differences attributed to deafness. Sign language use makes unique motor, visuospatial, and temporal demands on the brain (Campbell et al., 2008; MacSweeney et al., 2008). Indeed, our results from the ANOVA revealed a main effect of language experience in numerous brain areas, notably in the right hemisphere, where native users of ASL (deaf and hearing together) had more GMV than native users of English (deaf and hearing), whereas there were no areas that showed less GMV for native signers. These observations, especially the most robust finding in right middle frontal (BA9) gyrus, fit with the idea that spatial demands of ASL affect right hemisphere brain anatomy, here found in regions known to subserve planning of motor movements.

Our central question was how such effects of ASL experience would compound with deafness. The interaction analysis between language and sensory experience confirmed our prediction that some anatomical differences attributed to deafness are unique to native users of ASL. These included less GMV in right precentral gyrus (BA6) and insula (BA13) and more in right medial frontal gyrus (BA9) and left caudate in deaf native signers. The frontal locations imply a role for movement sequencing or 
Table 3. MNI coordinates of maxima of volume differences for whole-brain ANOVA

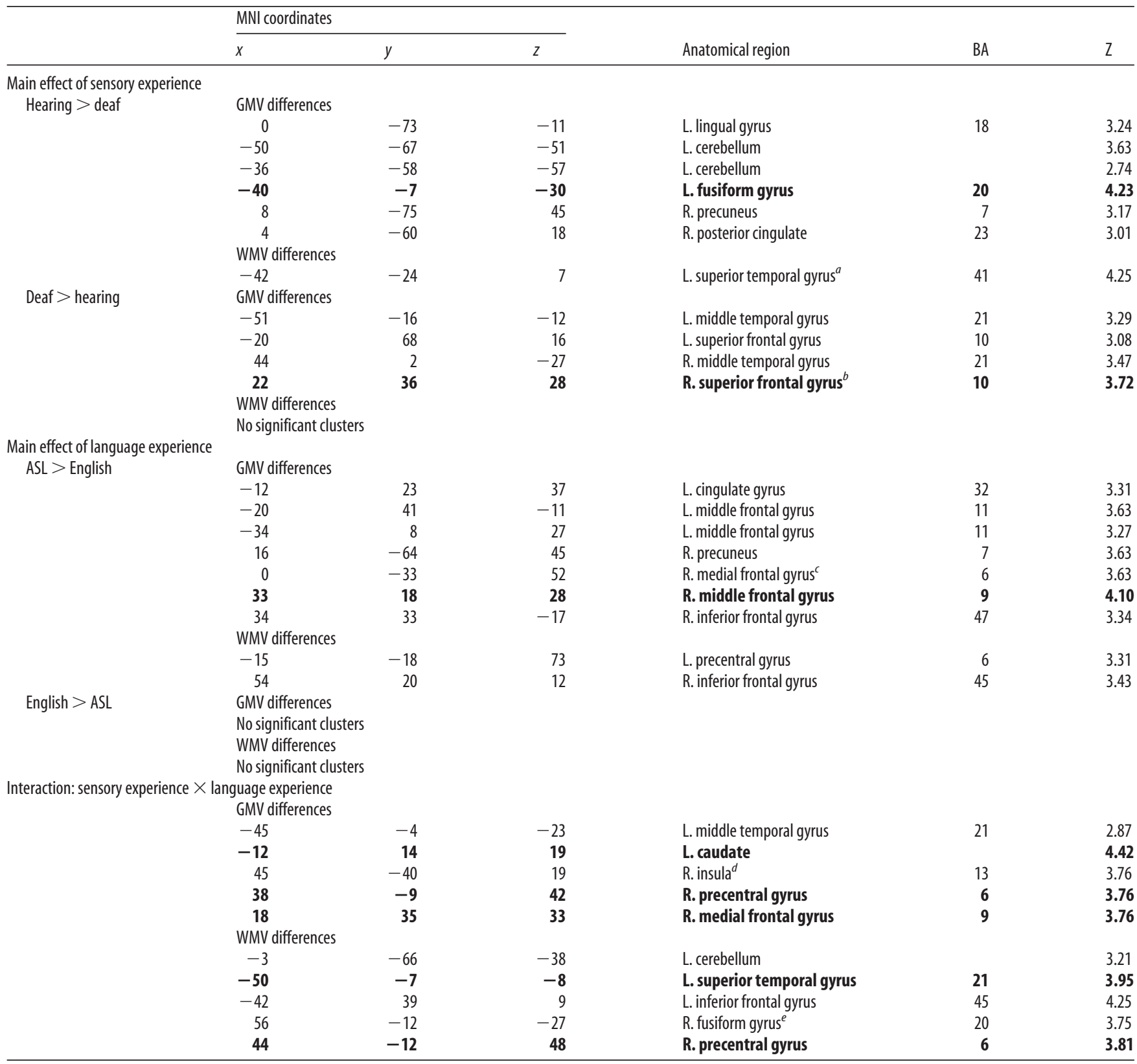

Clusters surviving a nonstationary correction at $p<0.05$ are listed in boldface.

${ }^{a}$ Cluster extends into the transverse temporal gyrus.

${ }^{b}$ Cluster extends into the middle and medial frontal gyri.

'Cluster extends into the paracentral lobule.

${ }^{d}$ Cluster extends into the supramarginal gyrus.

${ }^{e}$ Cluster extends into the inferior and middle temporal gyri.

decisions about actions in the absence of audition in signers. Notably, there was less WMV in the deaf native signers in left superior (BA21) and inferior frontal gyri (BA45), suggesting an impact on language areas in the absence of audition in signers. Together, these observations illustrate that some anatomical differences attributed to deafness are unique to native users of ASL. Native users of English in turn exhibit differential effects in other brain regions.

So, what are the most salient differences between deaf and hearing people independent of their language experience? The main effect of sensory experience revealed less GMV, most notably in fusiform gyrus (BA20). This focus, along with a similar finding in left lingual gyrus (BA18), suggests that visual areas within and outside of striate cortex contain less GMV in the deaf independent of their language experience. We also found more GMV in the deaf in right superior frontal gyrus (BA10), consistent with a report of "hypertrophy" in deaf signers in nearby prefrontal lobe structures (Leporé et al., 2010). This suggests the possibility of altered, perhaps enhanced executive function in all deaf in the absence of auditory information.

Our prediction was that brain matter within and surrounding auditory regions would be affected by deafness. Although we did not find overall less GMV in auditory cortex of the deaf, WMV was less in left superior temporal gyrus (BA41) in the deaf (native 
users of english and ASL combined) compared with hearing groups. This is consistent with prior studies of the deaf (Emmorey et al., 2003; Shibata, 2007; Kim et al., 2009; Leporé et al., 2010; Smith et al., 2011; Pénicaud et al., 2012) and our own betweengroup comparison presented in the first analysis. White matter reduction in auditory regions has been attributed to reduced myelination or increased pruning of auditory fibers, and thus fewer connections between auditory cortex and other brain regions (Emmorey et al., 2003), or as a result of functional reorganization (Finney et al., 2001; Bavelier and Neville, 2002; Fine et al., 2005). Indeed, there are reports of reduced white matter microstructural integrity measured with diffusion tensor imaging in superior temporal areas in adults with congenital hearing loss (Kim et al., 2009; Li et al., 2012). The interpretation of GMV differences is not straightforward. Much of the cortex undergoes thinning over development perhaps due to neuronal pruning (Giedd and Rapoport, 2010), but perisylvian language areas thicken (Sowell et al., 2004). Therefore, observation of more GMV could reflect normal maturation, but also failure to mature, depending on the location. In sum, the mechanisms underlying microstructural changes and their interpretation require further study and will benefit from other measures, such as gyrification patterns (Im et al., 2010).

Whether classical language areas in the left superior temporal lobe, also closely located to auditory cortex, are used differently in the deaf has been a topic of debate, especially in the context of sign language processing. Some have shown that sign language elicits activity in auditory regions (Nishimura et al., 1999; Lambertz et al., 2005; MacSweeney et al., 2008), whereas others have found activity in visual (but not auditory) cortex (Leonard et al., 2012). A recent report attributed activity in the left superior temporal sulcus in deaf signers to the linguistic (not visual sensory) aspects of sign language (Cardin et al., 2013). Our findings provide important new context for these observations, demonstrating that all deaf people in our study, independent of their native language background, have less white matter within left hemisphere auditory regions (BA41). However, the interaction analysis revealed critical new information: white matter in areas associated with language - the left STG (BA21) and inferior frontal gyrus (BA45) - is reduced only in deaf native users of sign (not in native users of English). Together, these findings provide new insights, inferring fewer white matter connections in early auditory regions in the deaf population in general, with extension of these WMV differences into areas known to process phonological information only in the deaf subjects who did not grow up with English as a native language. This may reflect a distinction that exists for phonology in ASL versus phonology in English.

Together, these results demonstrate that language background plays an important role in determining how deafness affects brain anatomy. Some anatomical differences attributed to deafness are unique to native users of ASL and are not generalizable to the larger deaf population. Further, the differential effects on brain areas subserving audition versus those subserving language can be disambiguated by studying deaf groups with distinct native language experiences, in this case English and ASL.

\section{References}

Allen JS, Emmorey K, Bruss J, Damasio H (2008) Morphology of the insula in relation to hearing status and sign language experience. J Neurosci 28:11900-11905. CrossRef Medline

Allen JS, Emmorey K, Bruss J, Damasio H (2013) Neuroanatomical differences in visual, motor, and language cortices between congenitally deaf signers, hearing signers, and hearing non-signers. Front Neuroanat 7:26. CrossRef Medline
Ashburner J (2007) A fast diffeomorphic image registration algorithm. Neuroimage 38:95-113. CrossRef Medline

Ashburner J, Friston KJ (2000) Voxel-based morphometry-the methods. Neuroimage 11:805-821. CrossRef Medline

Ashburner J, Friston KJ (2005) Unified segmentation. Neuroimage 26:839851. CrossRef Medline

Bavelier D, Neville HJ (2002) Cross-modal plasticity: where and how? Nat Rev Neurosci 3:443-452. CrossRef Medline

Bridge H, Cowey A, Ragge N, Watkins K (2009) Imaging studies in congenital anophthalmia reveal preservation of brain architecture in "visual" cortex. Brain 132:3467-3480. CrossRef Medline

Campbell R, MacSweeney M, Waters D (2008) Sign language and the brain: a review. J Deaf Stud Deaf Educ 13:3-20. CrossRef Medline

Cardin V, Orfanidou E, Rönnberg J, Capek CM, Rudner M, Woll B (2013) Dissociating cognitive and sensory neural plasticity in human superior temporal cortex. Nat Commun 4:1473. CrossRef Medline

Carreiras M, Seghier ML, Baquero S, Estévez A, Lozano A, Devlin JT, Price CJ (2009) An anatomical signature for literacy. Nature 461:983-986. CrossRef Medline

Emmorey K, Allen JS, Bruss J, Schenker N, Damasio H (2003) A morphometric analysis of auditory brain regions in congenitally deaf adults. Proc Natl Acad Sci U S A 100:10049-10054. CrossRef Medline

Fine I, Finney EM, Boynton GM, Dobkins KR (2005) Comparing the effects of auditory deprivation and sign language within the auditory and visual cortex. J Cogn Neurosci 17:1621-1637. CrossRef Medline

Finney EM, Fine I, Dobkins KR (2001) Visual stimuli activate auditory cortex in the deaf. Nat Neurosci 4:1171-1173. CrossRef Medline

Gallaudet Research Institute (2010) Regional and national summary report of data from the 2009-2010 annual survey of deaf and hard of hearing children and youth. Washington, DC: Gallaudet University.

Giedd JN, Rapoport JL (2010) Structural MRI of pediatric brain development: what have we learned and where are we going? Neuron 67:728-734. CrossRef Medline

Hayasaka S, Phan KL, Liberzon I, Worsley KJ, Nichols TE (2004) Nonstationary cluster-size inference with random field and permutation methods. Neuroimage 22:676-687. CrossRef Medline

Im K, Jo HJ, Mangin JF, Evans AC, Kim SI, Lee JM (2010) Spatial distribution of deep sulcal landmarks and hemispherical asymmetry on the cortical surface. Cereb Cortex 20:602-611. CrossRef Medline

Kara A, Hakan Ozturk A, Kurtoglu Z, Umit Talas D, Aktekin M, Saygili M, Kanik A (2006) Morphometric comparison of the human corpus callosum in deaf and hearing subjects: an MRI study. J Neuroradiol 33:158163. CrossRef Medline

Kim DJ, Park SY, Kim J, Lee DH, Park HJ (2009) Alterations of white matter diffusion anisotropy in early deafness. Neuroreport 20:1032-1036. CrossRef Medline

Lambertz N, Gizewski ER, de Greiff A, Forsting M (2005) Cross-modal plasticity in deaf subjects dependent on the extent of hearing loss. Brain Res Cogn Brain Res 25:884-890. CrossRef Medline

Leonard MK, Ferjan Ramirez N, Torres C, Travis KE, Hatrak M, Mayberry RI, Halgren E (2012) Signed words in the congenitally deaf evoke typical late lexicosemantic responses with no early visual responses in left superior temporal cortex. J Neurosci 32:9700-9705. CrossRef Medline

Leporé N, Vachon P, Leporé F, Chou YY, Voss P, Brun CC, Lee AD, Toga AW, Thompson PM (2010) 3D mapping of brain differences in native signing congenitally and prelingually deaf subjects. Hum Brain Mapp 31:970978. CrossRef Medline

Li Y, Ding G, Booth JR, Huang R, Lv Y, Zang Y, He Y, Peng D (2012) Sensitive period for white-matter connectivity of superior temporal cortex in deaf people. Hum Brain Mapp 33:349-359. CrossRef Medline

MacSweeney M, Capek CM, Campbell R, Woll B (2008) The signing brain: the neurobiology of sign language. Trends Cogn Sci 12:432-440. CrossRef Medline

Maguire EA, Gadian DG, Johnsrude IS, Good CD, Ashburner J, Frackowiak RS, Frith CD (2000) Navigation-related structural change in the hippocampi of taxi drivers. Proc Natl Acad Sci U S A 97:4398-4403. CrossRef Medline

Mather N, Hammill DD, Allen EA, Roberts R (2004) Test of silent word reading fluency. Austin, TX: Pro-Ed.

Mitchell RE, Karchmer MA (2004) Chasing the mythical ten percent: Pa- 
rental hearing status of deaf and hard of hearing students in the United States. Sign Language Studies 4:138-163. CrossRef

Nishimura H, Hashikawa K, Doi K, Iwaki T, Watanabe Y, Kusuoka H, Nishimura T, Kubo T (1999) Sign language "heard" in the auditory cortex. Nature 397:116. CrossRef Medline

Noppeney U (2007) The effects of visual deprivation on functional and structural organization of the human brain. Neurosci Biobehav Rev 31: 1169-1180. CrossRef Medline

Noppeney U, Friston KJ, Ashburner J, Frackowiak R, Price CJ (2005) Early visual deprivation induces structural plasticity in gray and white matter. Curr Biol 15:R488-R490. CrossRef Medline

Oldfield RC (1971) The assessment and analysis of handedness: the Edinburgh inventory. Neuropsychologia 9:97-113. CrossRef Medline

Peelle JE, Cusack R, Henson RN (2012) Adjusting for global effects in voxelbased morphometry: Gray matter decline in normal aging. Neuroimage 60:1503-1516. CrossRef Medline

Penhune VB, Cismaru R, Dorsaint-Pierre R, Petitto LA, Zatorre RJ (2003) The morphometry of auditory cortex in the congenitally deaf measured using MRI. Neuroimage 20:1215-1225. CrossRef Medline
Pénicaud S, Klein D, Zatorre RJ, Chen JK, Witcher P, Hyde K, Mayberry RI (2012) Structural brain changes linked to delayed first language acquisition in congenitally deaf individuals. Neuroimage 66C:42-49. CrossRef Medline

Ptito M, Schneider FC, Paulson OB, Kupers R (2008) Alterations of the visual pathways in congenital blindness. Exp Brain Res 187:41-49. CrossRef Medline

Shibata DK (2007) Differences in brain structure in deaf persons on MR imaging studied with voxel-based morphometry. Am J Neuroradiol 28: 243-249. Medline

Smith KM, Mecoli MD, Altaye M, Komlos M, Maitra R, Eaton KP, Egelhoff JC, Holland SK (2011) Morphometric differences in the Heschl's gyrus of hearing impaired and normal hearing infants. Cereb Cortex 21:991998. CrossRef Medline

Sowell ER, Thompson PM, Leonard CM, Welcome SE, Kan E, Toga AW (2004) Longitudinal mapping of cortical thickness and brain growth in normal children. J Neurosci 24:8223-8231. CrossRef Medline

Wechsler D (1999) Wechsler abbreviated scale of intelligence (WASI). San Antonio, TX: Psychological. 